\title{
Electrodynamics of quasi-two-dimensional BEDT-TTF charge transfer salts
}

\author{
Stephen Hill ${ }^{\dagger}$ \\ Department of Physics, Montana State University, Bozeman, MT 59717
}

(November 1, 2018)

\begin{abstract}
We consider the millimeter-wave electrodynamics specific to quasi-two-dimensional conductors and superconductors based on the organic donor molecule BEDT-TTF. Using realistic physical parameters, we examine the current polarizations that result for different oscillating $(\mathrm{GHz})$ electric and magnetic field polarizations. We show that, in general, it is possible to discriminate between effects (dissipation and dispersion) due to in-plane and interlayer ac currents. However, we also show that it is not possible to selectively probe any single component of the in-plane conductivity tensor, and that excitation of interlayer currents is strongly influenced by the sample geometry and the electromagnetic field polarization.
\end{abstract}

This brief report has been motivated by a number of recent studies of the millimeter-wave electrodynamics of quasi-two-dimensional (Q2D) organic conductors and superconductors based on the organic donor molecule BEDT-TTF (his-ethylenedithiotetrathiafulvalene, or ET for short). 1, 3, 4, 5.6 In recent years, measurements of the microwave surface impedance of high temperature superconductors (HTS) have played an important role in determining whether the superconducting gap function possesses nodes at certain points, or along certain lines in reciprocal space. Indeed, microwave penetration depth measurements are often cited as one of the key pieces of evidence sppporting a $d$-wave scenario in the hole-doped HTS. 8 Similar studies have atso been published for several organic superconductors, 10 though the symmetry of the superconducting state in these lower $\mathrm{T}_{c}$ compounds remains the subject of considerable debate 10 Aside from studies of superconductivity, millimeter-wave spectroscopies have also played a vital role in furthering our understanding of the often unystnormal state properties of many organic conductors. 3., 4,1$]$

Experiments usually involve placing a tiny single crystal at various locations within either rectangular or cylindrical enclosed cavities; 12,13 the linear dimensions of a typical sample are $\sim 0.5-2 \mathrm{~mm}$ parallel to the conducting planes and $\sim 0.05-0.5 \mathrm{~mm}$ in the perpendicular direction. In principle, dissipation due to the sample may be determined by measuring the change in dissipation (i.e. change in the resonance $Q$-factor) within the cavity upon insertion of the sample, while the penetration depth is related to the change in the central resonance frequency $\left(f_{o}\right)$ of the cavity upon insertion of the sample. In practice, this rigorous approach is rarely followed; rather, changes in dissipation and dispersion are recorded whilst varying some other external parameter, e.g. magnetic field, temperature, etc. The latter approach is particularly well suited to masonnetic resonance experiments, eso cyclotron resonance, 细 Josephson plasma resonance 6 , etc., where one is not so interested in the absolute value of the power dissipation within the cavity, but rather in the magnetic field strength at which maximum dissipa- tion occurs. Furthermore, one can perform fairly reliable lineshape analysis in this way, provided suitable care is taken to truly separate dissipative and dispersive contributions to the measurement.13

The high degree of anisotropy in the Q2D ET salts results in a situation in which the in-plane and interlayer electrodynamics differ considerably, particularly at low temperatures; by in-plane and interlayer electrodynamics, we mean the dynamics of in-plane and interlayer ac currents. These differences are attributable to the 3 to 4 orders of magnitude difference in conductivity parallel $\left(\sigma_{\|}\right)$and perpendicular $\left(\sigma_{\perp} \ll \sigma_{\|}\right)$to the conducting layers, as discussed at length in ref. [11]. In this paper, we consider the experimental geometries employed by several groups and, in each case, we discuss contributions to the electrodynamics due to both in-plane and interlayer currents. Before proceeding, we wish to correct a common misconception concerning the response of an anisotropic (super-) conductor to different electromagnetic field polarizations; namely that it is the polarizations of the induced currents that govern the electrodynamics, not the polarizations of the electromagnetic fields. We specifically examine the current polarizations arising in Q2D conducting ET salts for various ac electric or magnetic field polarizations.

Case I - in-plane measurements. The most obvious situation involves placing a sample within an oscillating electric field $(\tilde{\mathbf{E}})$ with polarization parallel to its highly conducting layers. Under these conditions, it is actually the displacement current $\partial \tilde{\mathbf{D}} / \partial t$ in the cavity that is responsible for driving currents within the sample (this is discussed further below). At liquid helium temperatures, the skin/penetration depths $\left(\delta_{\|}, \lambda_{\|}\right)$for in-plane currents in the normal/superconducting states range from about 0.2 to $5 \mu \mathrm{m}$ in high quality Q2D conducting ET salts. Thus, for this geometry, in-plane currents flow within a thin layer at the surface of the sample a distance $\delta_{\|}$or $\lambda_{\|}$from the sample's edges and faces, as illustrated in Fig. 1a. However, the shape of a typical Q2D ET salt is incompatible with the axial symmetry associated with such an electric field, i.e. single crystal samples generally form as thin platelets with the low conductivity direction 
perpendicular to the plane of the sample (see Fig. 1). Furthermore, such an electric field is also incompatible with the axial symmetry associated with the Q2D conductivity tensor, i.e. $\sigma_{\|}$is approximately isotropic within the planes, while $\sigma_{\perp}$ is considerably lower perpendicular to the planes. Consequently, an ac electric field parallel to the layers will become disrupted in the vicinity of the sample and may distort the flow of currents within the sample to such an extent that interlayer currents are also excited (not shown in Fig. 1a).

A better solution for in-plane measurements involves placing the sample within an oscillating magnetic field $(\tilde{\mathbf{H}})$ with polarization perpendicular to its highly conducting layers. Although this produces circulating currents, they are confined to within the highly conducting layers (see Fig. 1b) and, thus, one only has to consider inplane electrodynamics. Once again, these currents only flow within a distance $\delta_{\|}$or $\lambda_{\|}$of the sample's edges and faces. Fringing fields associated with the demagnetization of the interior of the sample produce negligible interlayer currents; this has been verified experimentally 13 and is discussed further below. Although this second method is preferable (Fig. 1b), both configurations (Figs. $1 \mathrm{a}$ and $\mathrm{b}$ ) reliably probe the in-plane electrodynamics of Q2D (super-) conducting ET salts. However, the conductivity $\sigma_{\|}$(or $\left.\delta_{\|}, \lambda_{\|}\right)$measured by either method represents an average over all in-plane directions, i.e. it is impossible to measure a single component of the in-plane conductivity tensor.

Case II - interlayer measurements. This situation is far more complex than the in-plane case. In particular, the electrodynamics differ considerably depending on whether the interlayer skin/penetration depth $\left(\delta_{\perp}, \lambda_{\perp}\right)$ is smaller (skin-depth regime) or larger (depolarization regime) than the linear dimension $(L)$ of the sample parallel to the conducting layers. In the latter case, the simplest experimental configuration involves placing the sample within an oscillating electric field $(\tilde{\mathbf{E}})$ with its polarization perpendicular to the highly conducting layers (see Fig. 1c). Under such circumstances, the $\tilde{\mathbf{E}}$-field penetrates the sample uniformly and drives bulk interlayer ac currents between the flat platelet surfaces; dissipation is then due entirely to $\sigma_{\perp}$.

The above situation is rarely, or never, realized at liquid helium temperatures in typical conducting ET salts - the reason is because $\delta_{\perp}$ and $\lambda_{\perp}$ rarely exceed about $200 \mu \mathrm{m}$ (usually $<100 \mu \mathrm{m}$ ). Consequently, even for the smallest samples studied, any induced interlayer currents will attenuate appreciably from the edges towards the center of the sample. If we assume a quasi-static approximation, which is justified on the grounds that the radiation wavelength (several millimeters) is larger than a typical sample, it becomes apparent that there is no ac electric or magnetic field polarization that can excite pure interlayer currents. To illustrate what happens in the skin-depth regime we first consider the case with the ac electric field $(\tilde{\mathbf{E}})$ polarization perpendicular to the conducting layers. Assuming $\delta_{\perp} \approx L / 10$, which is typical of many of the published studies, screening of the electric field within the bulk of the sample requires a uniform surface charge density to alternately flow between the large flat faces of the sample, as illustrated in Fig. 2. The only way that this charge can complete the circuit from one face to the other is via the edges of the sample, i.e. within a distance $\delta_{\perp}$ or $\lambda_{\perp}$ of the perimeter of the platelet. Consequently, currents must flow across the flat sample faces (i.e. parallel to the high conductivity planes) in order to maintain the uniform surface charge density necessary to screen the electric field from within the interior of the sample. These in-plane currents will be confined to a depth $\delta_{\|}$or $\lambda_{\|}$beneath the flat sample surfaces (Fig. 2). One can think of this current flow as a continuation of the displacement currents surrounding the sample; these, in turn, complete a circuit with the surface currents in the walls of the cavity.

One other way to generate interlayer currents is to place the sample within an oscillating magnetic field $(\tilde{\mathbf{H}})$ with its polarization parallel to the highly conducting layers (see Fig. 3). In spite of the conflicting symmetries associated with this field and the Q2D electronic system, this configuration seems to be the most widely used for studies of Q2D conducting ET salts.14 As in the previous case (Fig. 2), a combination of interlayer and in-plane currents are necessary to screen the oscillatory $\tilde{\mathbf{H}}$-field from within the sample. This time, the currents complete a circuit within the sample (i.e. there is no displacement current) that curl in a left-handed sense around the oscillatory $\tilde{\mathbf{H}}$-field. In-plane currents flow in opposite directions across the flat sample faces within a surface layer of thickness $\delta_{\|}$or $\lambda_{\|}$. Meanwhile, interlayer currents complete the circuit at the sample edges within a surface layer of thickness $\delta_{\perp}$ or $\lambda_{\perp}$. This situation is depicted in Fig. 3b.

Next we consider the relative contributions to the dissipation within the sample due to interlayer and in-plane currents for case II above (Figs. 2 and 3). In the skindepth regime, dissipation is governed by surface resistance $\mathbf{R}_{S}$, i.e. the real part of the surface impedance

$$
\hat{\mathbf{Z}}_{S}=\mathbf{R}_{S}+i \mathbf{X}_{S}=\sqrt{\frac{i \mu_{\mathrm{o}} \omega}{\sigma_{1}-i \sigma_{2}}}
$$

Provided one is not in the anomalous skin effect regime $(\delta \ll$ mean free path), which is never the case in even the best quality samples, 5 the above expression may be expanded assuming a Drude form for the conductivity. 12 In doing so, one finds two solutions for $\mathbf{R}_{S}$,

$$
\mathbf{R}_{S}=\frac{\mu_{o} \omega \delta_{\mathrm{o}}}{2}\left(1-\frac{\omega \tau}{2}+\frac{(\omega \tau)^{2}}{8}+\ldots\right)
$$

and

$$
\mathbf{R}_{S}=\frac{\mu_{o} \omega \delta_{\mathrm{o}}}{2}\left(\frac{1}{\sqrt{\omega \tau}}-\frac{1}{2(2 \omega \tau)^{5 / 2}}+\ldots\right)
$$


depending on whether i) $\omega \tau<1$ [Eq. (2), Hagen-Rubens regime, $\sigma_{1}>\sigma_{2}$ ], or ii) $\omega \tau>1$ [Eq. (3), Relaxation regime, $\left.\sigma_{2}>\sigma_{1}\right] ; \delta_{o}$ is the penetration depth obtained from the dc conductivity, i.e. $\delta_{o}=\left(\sigma_{d c} \omega \mu_{o} / 2\right)^{-0.5}$. Upon application of a dc magnetic field, the $\omega \tau$ product may be replaced by $\left(\omega-\omega_{c}\right) \tau$, where $\omega_{c}$ is the cyclotron frequency $\left(=e B / m^{*}\right)$. In general, the most relevant case to compare with published low temperature data is the latter (relaxation regime), though this situation is complicated when a dc magnetic field is included. 1 In either case, however, $\mathbf{R}_{S}$ is proportional to $\delta_{o}$. Thus, in the normal metallic state, dissipation due to the respective current polarizations will be proportional to the appropriate skin depth $\left(\delta_{\|}\right.$or $\left.\delta_{\perp}\right)$ multiplied by an appropriate area $\left(a_{\|}\right.$or $\left.a_{\perp}\right)$ for the surface across which that current flows. Then the ratio of the power dissipation due to interlayer $\left(P_{\perp}\right)$ and in-plane $\left(P_{\|}\right)$currents is given by $P_{\perp} / P_{\|}=a_{\perp} \delta_{\perp} / a_{\|} \delta_{\|}$.

The skin-depth ratio $\delta_{\perp} / \delta_{\|}$is a parameter which is often used as a measure of anisotropy, and in the case of the Q2D conducting ET salts ranges from about 30 to over 100. Thus, we see that interlayer currents will tend to dominate the measured dissipation, unless an extremely thin sample is used, i.e. area of faces exceeds the area of the edges by a compensating factor of 30 to 100 . In reality, the aspect ratio $a_{\|} / a_{\perp}$ is typically in the range 1 -5 for the samples used in most published studies (see e.g. $[2,4-6])$. Indeed, this is often cited as the reason why one can confidently attribute dissipation as being due entirely to $\sigma_{\perp}$ for measurements with $\tilde{\mathbf{H}}$ parallel to the conducting layers (again, see e.g. [2-6]). This has been confirmed by the few studies in which both the inplane (case I) and interlayer (case II) electrodynamics have been probed independently, e.g. in ref. [13], different cyclotron resonance lineshapes are observed for $\tilde{\mathbf{H}}$ parallel and perpendicular to the conducting layers which may be explained in terms of the different dominant current polarizations within the sample.

Things become more extreme in the superconducting phase, where the anisotropy parameter $\gamma\left(=\lambda_{\perp} / \lambda_{\|}\right)$is believed to exceed 100 (and maybe up to 200) in the most widely studied $\kappa$-phase ET salts. In this case, it is expected that $\sigma_{2} \gg \sigma_{1}$, and that $\mathbf{R}_{S}$ is given approximately by

$$
\mathbf{R}_{S}=\frac{\sigma_{1}}{2 \sigma_{2}} \sqrt{\frac{\mu_{o} \omega}{\sigma_{2}}}
$$

Matters become even more complicated in the presence of an external dc magnetic field, due to the creation of a mixed state. 16 The large $\gamma$ parameter results in a situation in which interlayer currents penetrate well into this mixed state. Consequently, one can expect the vortex structure/dynamics to have a major influence on $\sigma_{\perp}$. Indeed, the effects of vortices show up in the interlayer electrodynamics of the $10 \mathrm{~K}$ superconductor $\kappa-(\mathrm{ET})_{2} \mathrm{Cu}(\mathrm{NCS})_{2}$, when probed with $\tilde{\mathbf{H}}$ parallel to the conducting layers (Fig. 3) 1 a plasma mode is observed
- attributable to interlayer Josephson currents - which is not seen at all for $\tilde{\mathbf{H}}$ perpendicular to the layers. This confirms that, even within the superconducting phase, $\sigma_{\perp}$ dominates the dissipation in the second case (II) considered above, i.e. with $\tilde{\mathbf{H}}(\tilde{\mathbf{E}})$ parallel (perpendicular) to the layers.

Finally, we note that extreme caution should be exercised when comparing data for different electromagnetic field polarizations. In particular, although it is clear that $\sigma_{\perp}$ dominates the second case (II) considered above, it is apparent that different sample aspect ratios $a_{\|} / a_{\perp}$ are appropriate depending on whether $\tilde{\mathbf{H}}$ (Fig. 2) or $\tilde{\mathbf{E}}$-field (Fig. 3) excitation is used, and whether the sample is superconducting or not. Furthermore, for $\tilde{\mathbf{H}}$-field excitation, different aspect ratios will invariably apply for different polarizations within the conducting planes; this is the most probable explanation for the azimuthal polarization dependence observed in ref. [2].

In summary, using realistic sample parameters, we have examined the electrodynamics appropriate to Q2D conducting and superconducting ET salts, and for various different electromagnetic field polarizations. We find that it is possible to discriminate between the effects (dissipation and dispersion) of in-plane and interlayer ac currents. We also show that the ratios of the areas of different sample faces has a major bearing on the relative contributions of $\sigma_{\perp}$ and $\sigma_{\|}$to the dissipation.

We are indebted to Neil Harrison and Jochen Wosnitza for stimulating discussion. This work was supported by the Office of Naval Research (N00014-98-1-0538).

$\dagger$ email: hill@physics.montana.edu

${ }^{1}$ M. Dressel, S. Bruder, G. Grüner, K. D. Carlson, H. H. Wang and J. M. Williams, Phys. Rev. B 48, 9906 (1993).

2 J. M. Schrama, E. Rzepniewski, R. S. Edwards, J. Singleton, A. Ardavan, M. Kurmoo, and P. Day, Phys. Rev. Lett. 83, 3041 (1999).

${ }^{3}$ A. Ardavan, J. M. Schrama, S. J. Blundell, J. Singleton, and W. Hayes, Phys. Rev. Lett. 81, 713 (1998).

${ }^{4}$ S. Hill, J. S. Brooks, J. S. Qualls, T. Burgin, B. Fravel, L. K. Montgomery, J. Sarrao and Z. Fisk, Physica (Amsterdam) 246B-247B, 110 (1998).

${ }^{5}$ T. Shibauchi, M. Sato, A. Mashio, T. Tamegai, H. Mori, S. Tajima and S. Tanaka, Phys. Rev. B 55, R11977 (1997).

${ }^{6}$ M. Mola, S. Hill, J. T. King, C. P. McRaven, J. S. Qualls and J. S. Brooks, cond-mat/0001149.

${ }^{7}$ W. N. Hardy, D. A. Bonn, D. C. Morgan, R. Liang, and K. Zhang, Phys. Rev. Lett. 70, 3999 (1993).

${ }^{8}$ D. J. Van Harlingen, Rev. Mod. Phys. 67, 515 (1995).

${ }^{9}$ A. Carrington, I. J. Bonalde, R. Prozorov, R. W. Giannetta, A. M. Kini, J. Schlueter, H. H. Wang, U. Geiser, and J. M. Williams, Phys. Rev. Lett. 83, 4172 (1999).

${ }^{10}$ R. H. McKenzie, Science 278, 820 (1997). 
${ }^{11}$ Stephen Hill, Phys. Rev. B 55, 4931 (1997).

12 O. Klein, S. Donovan, M. Dressel and G. Grüner, Int. J. Infrared and Millimeter Waves 14, 2423 (1993); S. Donovan, O. Klein, M. Dressel, K. Holczer and G. Grüner, ibid. 14, 2459 (1993); M. Dressel, O. Klein, S. Donovan, G. Grüner, ibid. 14, 2489 (1993).

${ }^{13}$ M. Mola, S. Hill, P. Goy and M. Gross, Rev. Sci. Inst. 71, 186 (2000).

14 The reason for this is befanco most studies incorporate strong dc magnetic fields. 3.4 . 6 . This causes experimental design constraints which tend to preclude $\tilde{\mathbf{E}}$-field excitation of interlayer currents. 13

${ }^{15}$ It is well documented that interlayer transport in the Q2D conducting ET salts is on the verge of being incoherent; this is due to the extremely weak band dispersion in the interlayer direction. Thus, a carrier contributing to in-plane current flow (within a layer of thickness $\delta_{\|}$) will undergo many collisions before it leaves the skin layer, i.e. a carrier will typically only make it across a few conducting layers $\left(\ll \delta_{\|}\right)$between collisions. Meanwhile, $\delta_{\perp}$ is considerably greater than the mean free path in even the cleanest ET salts (mean free path $\sim 5 \mu \mathrm{m}$ ).

${ }^{16} \sigma_{1}$ and $\sigma_{2}$ vary dramatically in the vicinity of the resonance condition $\omega_{c}=\omega$, with $\sigma_{2}$ changing sign at $\omega_{c}=\omega$. This subject will be treated in detail in a future publication. 
a)
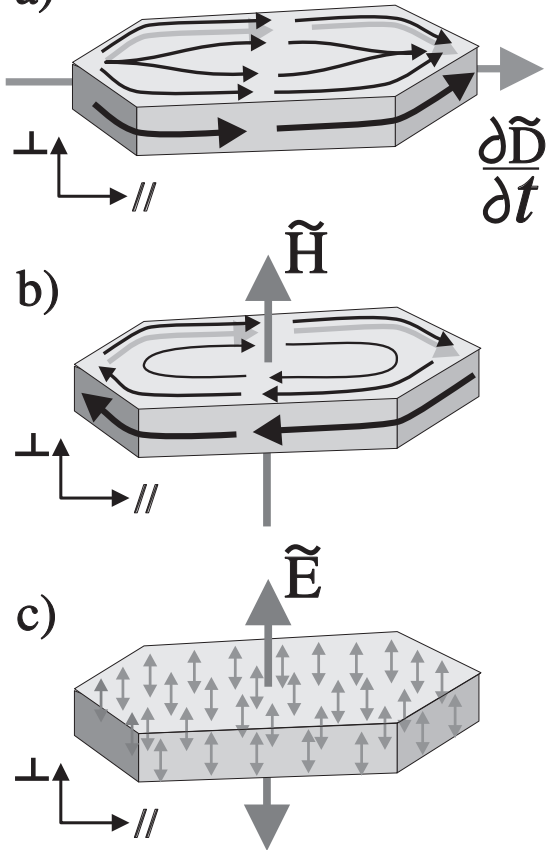

FIG. 1. Schematic representing the flow of in-plane currents (case I) for a) $\tilde{\mathbf{E}}$-field excitation, and b) $\tilde{\mathbf{H}}$-field excitation; in both a) and b), currents flow only within a distance $\delta_{\|}$or $\lambda_{\|}$(《 sample dimensions) of the sample surface. c) depicts uniform interlayer currents (case II) for a sample in the depolarization regime, driven by an $\tilde{\mathbf{E}}$-field perpendicular to the layers.
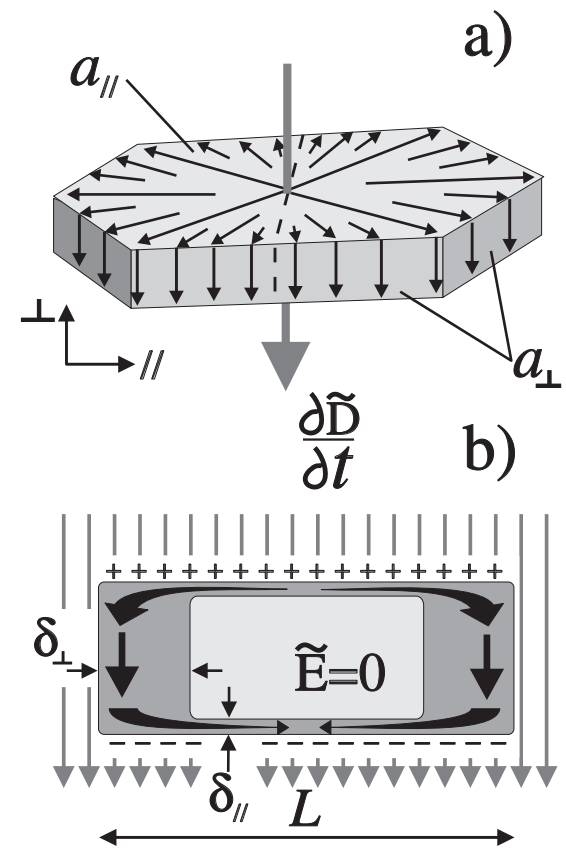

FIG. 2. $\tilde{\mathbf{E}}$-field excitation of interlayer currents (case II) for a sample in the skin-depth regime. The currents respond to the surrounding displacement current $\partial \tilde{\mathbf{D}} / \partial t$ in such a way as to maintain the necessary surface charge to screen the $\tilde{\mathbf{E}}$-field from within the sample. The dashed line in a) indicates the location of the cross-section shown in b). Note: both in-plane and interlayer currents are excited; the former flow within a surface layer of thickness $\delta_{\|}$parallel to the large sample faces, while the latter flow within a surface layer of thickness $\delta_{\perp}$ at the sample edges.

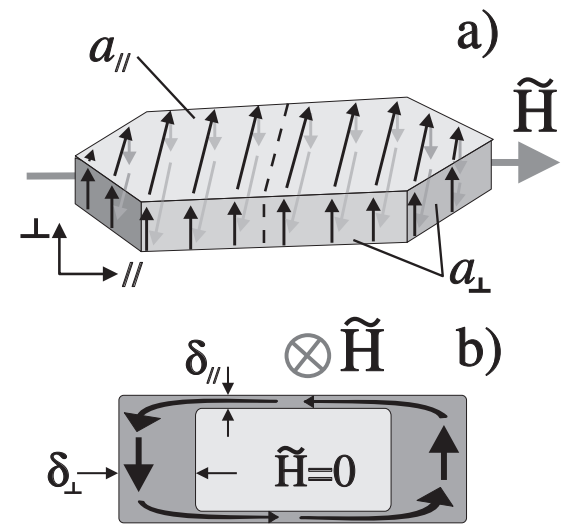

FIG. 3. $\tilde{\mathbf{H}}$-field excitation of interlayer currents (case II) for a sample in the skin-depth regime. The dashed line in a) indicates the location of the cross-section shown in b). Note: both in-plane and interlayer currents are excited; the former flow within a surface layer of thickness $\delta_{\|}$parallel to the large sample faces, while the latter flow within a surface layer of thickness $\delta_{\perp}$ at the sample edges. 